\title{
Les constructions siamoises en français et dans cinq autres langues européennes. Étude sur la comparative corrélative
}

\begin{abstract}
This article discusses the comparative correlative constructions in French and five other European languages. A comparative correlative construction is a binary structure whose two parts are conjoined as Siamese twins (to use the fine metaphor invented by Savelli, a French linguist). The most striking feature of this construction is the fronting of the comparatives in two clauses which are interdependent and inseparable, though at first glance no clear relation can be seen, neither in French nor in English. It is hoped that this study of six European languages will contribute to an elucidation of the structuring of the two clauses and the relation between the two parts as well as the meaning they convey.
\end{abstract}

\section{Introduction}

La comparative corrélative est une construction binaire dont les deux parties $(\mathrm{A}+\mathrm{B})$ sont unies comme les doigts de la main ou comme des soeurs siamoises, pour employer la belle métaphore utilisée par Savelli (1993). Dans sa thèse, Savelli se penche surtout sur la construction plus-plus. Or, les constructions en plus-moins, moins-mieux, meilleurmieux, plus-moindre etc. étant de même nature, je vais élargir le champ

\footnotetext{
* Lilian Stage

Copenhagen Business School

Department of International Language Studies and Computational Linguistics

Dalgas Have 15

DK-2000 Frederiksberg

ls.isv@cbs.dk
}

Hermes - Journal of Language and Communication Studies no 43-2009 
d'application de la métaphore ${ }^{1}$. Ce qui caractérise la construction siamoise, c'est que les deux parties $(\mathrm{A}+\mathrm{B})$ sont solidaires et inséparables sans que l'on puisse toujours à première vue établir une relation de dépendance syntaxique entre elles. Dans cet article, je tenterai de démontrer à l'aide d'une étude multicontrastive (de six langues européennes : l'allemand, l'anglais, le danois, l'espagnol, le français, et l'italien) qu'en dépit des apparences, il s'agit en français aussi d'une construction de subordination syntaxique. Comme l'indique déjà le titre, je me permettrai donc de mettre l'accent sur le français. Le trait le plus frappant de la construction siamoise, c'est le déplacement à gauche de deux comparatifs, qui sont placés en tête des deux séquences. La construction siamoise sert, sur le plan sémantico-pragmatique, à établir une relation entre deux mouvements ou deux variations exprimés par les deux comparatifs (augmentation et/ou diminution). Grâce à leur expressivité, les siamoises apparaissent à l'oral comme à l'écrit, dans les proverbes et les dictons, dans la publicité, dans la conversation courante et dans toutes sortes de genres écrits² :

Fidèle à l'équation " plus les gens achètent, mieux ils se portent ", Ikéa revendique sa politique d'achats impulsifs (Olivier Bailly et alii, Ikéa un modèle à démonter 2006)

La pub, plus c'est con, plus ça rentre dans le crâne. (France 2. 16-102002)

Moins une compétence est automatisée et plus elle nécessite d'attention et de place dans notre mémoire de travail, ajoute Jean-Pierre Jaffe, linguiste au CNRS (Le Nouvel Observateur 6-12 septembre 2007)

Ma mère, moins j'avais d'histoires avec elle, et mieux je me portais. (Philippe Djian, Frictions p. 12)

1 Mon corpus est constitué d'exemples que j'ai relevés au cours de mes lectures et d'exemples que j’ai trouvés dans Frantext et sur Google. Il va de soi que ce corpus me permet uniquement d'indiquer des tendances et non pas des certitudes statistiques quant à la fréquence de tel ou tel phénomène. Je tiens, cependant, à souligner que pour toutes les langues étudiées, j’ai passé en revue un très grand nombre d'exemples authentiques en faisant des recherches sur Google.

2 Dans les exemples cités, les syntagmes comparatifs sont en caractères gras. Le syntagme comparatif peut être continu ou discontinu. Voir sous 6. 
L'ennui, c'est que l'éthique, c'est un peu comme la culture : moins on en a, plus on l'étale - en parler trop est louche (Corinne Maier, Bonjour paresse p. 57)

Cadre est un titre, non une fonction. Mieux vaut l'être que ne pas l'être : comme tout le monde passe son temps à faire le travail de la personne du dessus, plus on est haut, moins on a à faire - plus vous êtes importants, moins vous travaillez, c'est une loi d'airain du monde du travail. (Corinne Maier, Bonjour paresse p. 69)

Minnie thinks perhaps everything will become clearer in due course, so she reads on, but the deeper she gets into the story the thicker is the fog of her incomprehension (David Lodge, Author, Author p.20)

The long first section about the hero's childhood stood up well on reacquaintance, but the further Henry read into the part of the novel which was new to him, the more his earlier misgivings were confirmed. (David Lodge, Author, Author p.141)

.... Falls das Lesen dieser Schicksale euch Tränen in die Augen treibt, dann geht es euch so wie mir und je öffter man sie liest desto mehr denkt man darüber nach. (www.kaninchenbande10.de/html/schicksalsschlage)

Je höher wir kamen, desto weiter wurde unser durch die Dunkelheit so begrenzter Gesichtskreis. (www.logoslibrary.eu)

Cuanto más pequeño es el niño, más difícil es separar los diferentes aspectos de su desarrollo: perceptivo, cognitivo, lingüístico y afectivo, aunque a partir de la edad preescolar es posible individualizar la afectividad y la inteligencia. (www.em-consulte.com)

Un problema cuanto más precisado está tanto más fácil es encontrar la solución, al contrario cuanto más difuso e impreciso más difícil es darle respuesta. ... (www.isftic.mepsyd.es/)

Quanto più lontano stanno dagli occhi, tanto più vicini al cuore sono i sentimenti che cerchiamo di soffocare e dimenticare.» Paulo Coelho ... (www.patriziolongo.com/citazione)

Quanto migliore è l'informazione fornita, tanto più il consumatore puó fidarsi nello scegliere il mutuo casa da contràrre. (www.europamica.it)

Pour bien cerner les caractéristiques syntaxiques de la construction 
siamoise, il faudrait étudier au moins les points suivants : introducteur, déplacement à gauche des comparatifs, structure binaire, césure, emploi de la conjonction et dans la césure, relation entre les séquences, éclatement du comparatif (i.e. syntagmes continus ou discontinus), place du sujet dans les deux séquences et emploi d'une siamoise dans la phrase averbale. Une étude multicontrastive devrait permettre de dégager les ressemblances et les différences entre les langues examinées et de pointer ainsi les domaines qui seraient susceptibles de poser problème aux professionnels qui doivent naviguer entre plusieurs langues. A la fin de l'article, on trouvera un tableau synoptique, montrant les différences et les ressemblances entre les six langues.

\section{Définition}

Les caractéristiques de la construction siamoise sont les suivantes :

- la construction est composée de deux séquences : A et B

- les deux séquences $\mathrm{A}$ et $\mathrm{B}$ sont solidaires et inséparables

- les deux séquences contiennent des comparatifs déplacés à gauche, en tête des séquences

- la construction exprime une covariation ${ }^{3}$ entre A et B

- la construction permet à l'interlocuteur d'inférer soit une relation conditionnelle soit une relation causale

Il est facile de prouver que la construction siamoise forme un tout organique, une unité dont les deux parties sont solidaires. La portée de la négation et de la question démontre sans conteste cette solidarité. La négation et la question ne peuvent porter que sur l'ensemble c'est-àdire sur la relation que le locuteur cherche à établir entre les deux séquences.

Il n'est pas vrai que plus on pense, moins on sent ; mais il est vrai que plus on juge, moins on aime. ... (www.citationsdumonde.com)

Est-il vrai que moins on fume plus c'est facile d'arrêter? (www.atoute.org)

3 Le terme est d'Abeille et de Borsley 2005. Voir plus loin sous 9. 
Et je pense que le plus tôt on claque la porte aux turcs le mieux. Je ne pense pas que plus on attend mieux on pourra le faire sans dégats. (www.blogdsk.net)

S’il est vrai que testa, la tête, est un pot, est-ce que plus on parle, plus on le vide, ou bien est-ce qu'on le remplit ? (perso.club-internet.fr)

Mais la facture est-elle liée aux délais ? Est-ce que plus on traîne, plus on paie ? (www.ecu-activities)

Il en est de même avec la question-tag n'est-ce pas qui souligne aussi l'ensemble. Dans l'exemple suivant, on pourrait sans problème ajouter n'est-ce pas.

Moins on sait qui on est, plus on est dogmatique et sectaire. (www. lejdd.fr/cmc/politique)

Moins on sait qui on est, plus on est dogmatique et sectaire, n'est-ce pas?

\section{Introducteur}

Alors que la construction siamoise est annoncée, dans la plupart des autres langues, par un mot introducteur, le français moderne n’a pas recours à un tel introducteur - dans la majorité des exemples relevés. C’est le comparatif déplacé à gauche qui 'signale’ la construction.

\begin{tabular}{|l|l|l|}
\hline Langue & Séquence A & Séquence B \\
\hline allemand & je & desto, um so $(j e)^{5}$ \\
\hline anglais & the & the \\
\hline danois & jo & jo, desto, des \\
\hline espagnol & cuanto $^{6}$ & tanto, - \\
\hline français & - & - \\
\hline italien & quanto, tanto, - & tanto, - \\
\hline
\end{tabular}

Tableau 1. Introducteurs

4 On trouve parfois um so dans la séquence A (pour les danophones voir Stage 2003 : 44).

5 Je est plutôt rare dans la séquence B (Voir Bech 1964 : 51).

6 Dans la langue orale, quotidienne, on trouve aussi mientras más... más. En Amérique latine, cette construcion est également courante dans la langue écrite : Mientras más plata tiene una mujer, menos come : la clase alta se distingue por la flacura (Isabel Allende, Mi país inventado p. 70). Voir Butt \& Benjamin 1988/98 :80). 
Comme il ressort du tableau ci-dessus, l'introducteur est soit identique dans les deux séquences (anglais, danois et italien) soit différent (allemand, danois, espagnol et italien). Soit il n'y en a pas (français). C'est en italien que la variation est la plus grande - théoriquement. En fait, l'introducteur reste très souvent inexprimé en italien comme en français.

Si les linguistes s'accordent pour voir dans l'introducteur de la séquence A une sorte de conjonction de subordination qui ne s'emploie que dans le cas des siamoises, ils ne se prononcent pas de façon claire sur l'introducteur de la séquence B. Il s'agit là, à mon avis, d'un adverbe qui résume la séquence $A$ comme on le voit très souvent dans les constructions conditionnelles dans toutes les langues examinées :

Si ton adversaire refuse alors la partie est finie et tu as gagné 1 point.

S'il accepte; alors le gagnant gagnera 2 points. (www.chez.com)

A propos du mot introducteur the en anglais, Jespersen (1924: 251) dit ceci: « in «the more, the merrier» and similar collocations of two members, the first the is relative, while the second the is demonstrative. The first member may be called the determinant, and the second the determined». En d'autres mots, la séquence A est à considérer, selon Jespersen, comme une subordonnée.

Frankly the more I hear about him, the less I like him. (David Lodge, A trilogy, Changing places p. 112)

L’on trouve aussi parfois des énoncés en anglais sans introducteur, mais les exemples relevés ne sont pas nombreux.

Something called duty. Old-fashioned word, these days. Longer you put it off, harder it'll be. Go home. They'll welcome you with open arms. (Le Carré, The Constant Gardener p. 325)

I'm the other way round. De-emotionalised. Un-emotionalised. Emotioned out. Less you feel, louder you yell. Bigger the vacuum you've got to fill. Not You. Me. (Le Carré, The Constant Gardener p. 502)

Bech (1964: 49) considère que je...umso/desto appartiennent aux conjonctions de subordination. Il est très rare, selon Bech, de trouver je dans la séquence B. Selon Helbig \& Buscha (1996:461), l'ensemble allemand je .... um so est une conjonction de subordination, une locution conjonctionnelle. En danois jo..../desto, jo est considéré par Han- 
sen (1967 : III, 275) comme un 'ensemble complexe' En italien et en espagnol, l'introducteur de la séquence A est un adverbe relatif et l'introducteur de B un adverbe quantitatif. Si l'introducteur est rare en français, il n'est toutefois pas totalement inconnu. Mais on le trouve surtout dans des textes anciens (avant 1900) et dans les langues régionales et populaires ${ }^{7}$. Mes recherches dans Frantext et sur Google dans des textes publiés après 1945 n'ont donné que quelques exemples avec tant plus ... tant plus, tant plus que ... tant plus que, d'autant plus ... d'autant plus, le plus ... le plus/le mieux ou au plus ... au plus. ${ }^{8}$

Au plus l'eau est chaude, au plus elle est tentante (Fr 2 - Chambéry, juillet 2005)

Au plus, vous montrerez des signes d'énervement, au plus votre bébé s'agitera...c'est un cercle vicieux. Distrayez-le ! (www.maman-nature.com)

Elle croit que tant plus que ça se voit, tant plus que c'est beau. (Frantext, Colette, Claudine à l'école/ 1900 p. 299)

Il est à noter qu'en français les introducteurs ne sont jamais des conjonctions de subordination ni des adverbes relatifs, mais des adverbes indiquant la quantité ou des superlatifs placés en tête de séquence. Il n’y a donc toujours rien qui permette de décider du statut syntaxique à donner à la séquence $\mathrm{A}$ en français.

\section{Structure binaire}

Comme il a été dit plus haut, ce qui caractérise de prime abord la construction siamoise, c'est le déplacement à gauche, en tête de séquence, de deux comparatifs appartenant à deux séquences consécutives. Selon Allaire (1982: 48) : « Il y a implication bilatérale des deux adverbes qui n'ayant aucune indépendance l'un par rapport à l'autre constituent l'une de ces unités textuelles que nous appelons syntagmes ». Et plus loin : " Nous avons vu que la présence de deux ou plusieurs adverbes est constitutive du syntagme » (1982: 359). La formule « implication bilatérale des deux adverbes " n'est pas heureuse puisqu'elle exclut

7 Voir Sandfeld 1965:451, Grevisse 1986:1440 et Savelli 1993:15 et 21.

8 Pour plus d'exemples voir Savelli 1993 : 13 et Lilian Stage 2003 : 41-42. 
les constructions siamoises introduites par les comparatifs synthétiques d'adjectifs tels que meilleur, moindre et pire..

Meilleur sera le vin, meilleur sera le civet. Le (www.cuniculture. info/)

Plus on s'éloigne du cœur de l'agglomération moindre est le service rendu par le réseau de transport en commun : faible maillage, faible fréquence. ... (www.humanite.fr/2005)

Pire sera la situation en 2007 et mieux cela sera pour eux, pensent-ils ... (forum.framasoft.org)

Il serait préférable de parler d' 'implication bilatérale de deux comparatifs en position initiale'. La structure fondamentale est une structure binaire simple constituée de deux séquences A + B :

Plus il fait chaud... mieux ça pousse ! (www.passionbio.com)

Ceux qui ont eu la chance de rencontrer des modèles de courage, d'invention, d'optimisme ont pu se constituer des croyances dynamisantes - exemple : «plus un défi est difficile, plus il est excitant » ou " en cas de besoin, je sais comment obtenir de l'aide ». (Le Nouvel Observateur 8-14 janvier 2004 p. 6)

Parfois on trouve plusieurs constructions siamoises simples $(\mathrm{A}+\mathrm{B})$

séparées par des points virgules ou des virgules.

Plus les enseignants souffrent dans leur pratique professionnelle devenue « impossible », plus ils se crispent; plus ils se crispent, moins ça change ; moins ça change, plus ils se désespèrent (Monde de l'Education juillet-août 2005 p. 87)

La domination vécue évide la vie de l'intérieur, n’en laisse plus subsister que l'apparence. Tout se concentre sur la forme, la peau, comme si l'individu était statufié et privé d'intériorité. Il n'est jamais que ce que le regard du dominant fait de lui. On le voit, mais plus on le voit et moins on le voit, plus il est visible et plus il est invisible, plus il occupe le champ de vision des « autres », moins il a de réalité personnelle. Il est une forme creuse. (Blanchard, Bancel et Lemaire, La fracture coloniale p. 217)

On trouve aussi, surtout dans la langue écrite, des structures binaires complexes comme dans les exemples suivants où $\mid$ marque la césure : 
Plus il se sent en sécurité, plus il est attaché, | mieux il explore son monde. (Le Nouvel Observateur 3 janvier 2005 p. 13)

Avec le financement des 35h, plus on boira, plus on fumera, | moins on devra travailler; (membres.lycos.fr)

Plus elle paraît fondée, plus elle est étayée par la logique, | plus elle court le risque d'être démentie. (Frantext, Dutourd, Jean /Pluche ou l'amour de l'art/1967 p. 253)

Comme le montre Georges Mauco, plus l’industrie est récente, plus elle est concentrée, plus les conditions de travail y sont difficiles, | et plus on y trouve d'immigrés (Noiriel, Etat, nation et immigration p. 111)

Une histoire d'autant plus difficile à saisir dans le cas de l'immigration que plus l'agression xénophobe a été violente, plus les conflits ont été intenses, | et plus les efforts « compensateurs » visant à effacer les différences ont été importants. (Noiriel, Etat, nation et immigration p. 130)

Plus les citoyens ont été confrontés dans leur vie quotidienne avec elle [l'infrastructure communicationelle], plus ils se sont familiarisés avec elle, | plus cela a consolidé le sentiment d'appartenance nationale (Gérard Noiriel, Etat, nation et immigration p. 200)

Dans ce type d'énoncés, il peut y avoir jusqu'à trois ou quatre séquences introduites par des comparatifs déplacés à gauche, mais les séquences se répartissent toujours, à mon avis, en deux parties selon le schéma ci-dessous avec ce que j'appellerai une 'césure' entre elles. La césure est une 'coupure sémantico-syntaxique qui marque la transition vers la conclusion’. Elle est signalée par le symbole |. Cette binarité fondamentale est commune aux six langues.

$$
\begin{aligned}
& A_{1}, A_{2} \mid B \\
& A_{1}, A_{2}, A_{3} \mid B \\
& A_{1}, A_{2}, A_{3}, A_{4} \mid B \\
& A \mid B_{1}, B_{2}
\end{aligned}
$$

La première partie de la siamoise peut renfermer trois ou quatre sousséquences, alors que la séquence $B$ n'en contient le plus souvent que deux. Théoriquement, il n'y a aucune limite quant au nombre de sousséquences dans les deux parties. C’est notre capacité à comprendre les 
énoncés complexes qui fixe la limite supérieure. Il se trouve que nous acceptons plus facilement un nombre élevé de sous-séquences en A que dans la conclusion en B (voir aussi les exemples cités ci-dessus).

Cuanto más mujeres conozco,| menos creo encontrar el ideal y más necesito hallar el ideal. (www.portaldel9.com.ar/)

The higher up the ladder people went in the conglomerate, Vic had observed,| the bigger their desks became and the less paper and other impedimenta they had on them. (David Lodge, A trilogy, Nice work p.880)

The more the men whistled, the more ribald their remarks, | the faster she walked; but the faster she walked, the more of a sexual object, or sexual quarry, she became, (...). (David Lodge, A trilogy p. 695)

Dans les langues autres que le français et l'anglais, la détermination de la césure repose sur des critères syntaxiques indiscutables. Le premier critère c'est le mot introducteur : l'allemand, le danois, l'espagnol et l'italien disposant d'introducteurs réservés soit à la séquence A soit à la séquence $\mathrm{B}$, il est le plus souvent facile de décider si, dans ces langues, on a affaire à des sous-séquences en $\mathrm{A}$ ou bien à des sous-séquences en B (voir tableau ci-dessus). Il faut remarquer qu'en danois moderne, on a tendance à utiliser le même introducteur (jo.. jo) dans les deux séquences et qu'en allemand on trouve aussi, quoique rarement, je dans la séquence B (voir Bech 1964 :51). Le deuxième critère c'est la conjonction de coordination (og, and, und et $y$ ), qui peut coordonner les sousséquences en danois, anglais, allemand et espagnol. Dans ces quatre langues, la conjonction de coordination (og, and, und et $y$ ) ne s'emploie jamais dans la césure. (voir plus loin sous 5.3)

Je größer die Toleranzgrenze, | um so kleiner wird die Falsche Rückweisungs-Rate und um so größer wird dafür die andere Fehlerwahrscheinlichkeit, die Falsche Akzeptanz-Rate. (www.darmstadt.gmd)

Jo mere engagerede og jo mere aktive kollegerne er, | desto større indflydelse kan man få. (Faglig håndbog 2000 p. 13)

Cuanto más cerca pase la órbita terrestre de alguna de ellas, y cuanto más cercanas en el tiempo sean estas, | mayor será, teóricamente, la tasa meteórica. (astroclub.net/mercure) 
E' altrettanto noto che quanto più alta è la temperatura e quanto più elevata è l'umidità dell'aria | tanto più si suda. (www.fids.it/Struttu$\mathrm{ra} /$ )

Dans les énoncés où l'introducteur de B est le même qu'en A (en allemand : je, en anglais : the, en danois : jo et en italien : tanto), la détermination de la césure pose les mêmes problèmes en anglais et en italien qu'en français, s’il faut tenir compte uniquement de critères syntaxiques pour fixer la césure. Par contre, en allemand et en danois (langues V2), il existe un autre critère syntaxique solide : l'inversion obligatoire du sujet de la séquence B. Dans les exemples suivants, le sujet inversé est mis en italique.

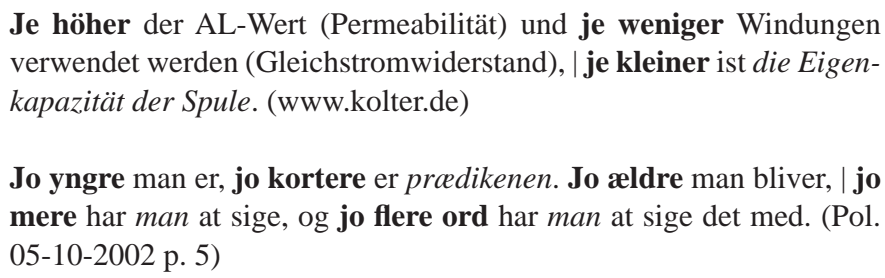

Je höher der AL-Wert (Permeabilität) und je weniger Windungen verwendet werden (Gleichstromwiderstand), | je kleiner ist die Eigenkapazität der Spule. (www.kolter.de)

Jo yngre man er, jo kortere er præedikenen. Jo ældre man bliver, | jo mere har man at sige, og jo flere ord har man at sige det med. (Pol. 05-10-2002 p. 5)

L'inversion du sujet non-clitique est possible en français, mais elle n'est jamais obligatoire. C'est pourquoi je distinguerai plus tard, dans le tableau synoptique, entre inversion syntaxique obligatoire et inversion stylistique facultative.

En anglais, même en anglais moderne, la césure est parfois signalée par l'inversion du sujet dans la séquence B. Mais l'ordre sujet-verbe y reste néanmoins l'ordre normal.

\begin{abstract}
Michael Reynolds was going insane slowly but inevitably insane. And the most terrible part of it was that he knew it. Since the last forced injection, there had been nothing he could do about the relentless onset of madness. The more he struggled to ignore the symptoms, the more acutely he became aware of them, | the deeper into his mind dug those fiendish chemical claws that were tearing his mind apart." (Alistair Maclean, The Last Frontier, Pbk. Bookseller Inventory)
\end{abstract}

En français, il est plus difficile de déterminer la césure comme le montre l'exemple suivant, apparemment assez simple :

Mieux on se connaît, mieux on s'accepte, | mieux on accepte les autres (toutsurdeneuve.free.fr) 
Où faut-il situer la césure en français dans des énoncés à plus de deux séquences ? Regardons l'énoncé suivant où on trouve quatre comparatifs en tête de séquence:

C'est alors que l'on voit se mettre en place une stratégie massive de communication où la machine publicitaire constitue un rideau de fumée chargé d’étouffer une réalité de plus en plus éloignée du développement durable. Plus le mensonge est gros, mieux il est empaqueté, plus il est répété, plus il a de chance de laisser des traces dans l'opinion. Cultivons l'hypocrisie, semons le doute, épandons le mensonge, cela servira d'engrais à nos profits! (www.developpement-durable.com)

Comme il n’y a pas de critères syntaxiques solides pour guider le récepteur, celui-ci est obligé de recourir à la sémantique et à sa connaissance du monde pour situer la césure et trouver le début de la conclusion. N’y aurait-il pas un critère phonétique pour guider le récepteur ? Selon Allaire (1982: 355) l'intonation pourrait en effet orienter le récepteur dans le processus d'interprétation. Mais ce critère n'est évidemment valable que pour les énoncés oraux. Pour les énoncés écrits, ce qui pose problème, c'est justement de comprendre où il faut situer la césure - donc de comprendre où commence la conclusion. C'est seulement quand l'énoncé aura été lu et interprété que le récepteur pourra opter pour une intonation toujours montante ou bien descendante. Si l'on n'avait sous les yeux que les deux premières séquences de l'énoncé précédent, celui-ci serait tout naturellement interprété comme une structure binaire simple en A + B :

Plus le mensonge est gros, | mieux il est empaqueté.

Dès que l'on poursuit la lecture, cette interprétation s'avère cependant impossible et on comprend que logiquement, il s'agit de trois sous-séquences en $A$ et de la conclusion en $B: A_{1} A_{2} A_{3} \mid B$. La révision de l'interprétation a certainement lieu si vite que le récepteur n'en est guère conscient. Pour les énoncés en français, le récepteur est donc obligé de s'appuyer sur la sémantique de chaque séquence et sur sa connaissance du monde pour fixer la césure.

Plus une université est récente (et a donc des taux d'encadrement plus faibles), plus une discipline est au bas des hiérarchies académiques, plus les origines sociales et solaires des étudiants y sont hétérogènes | et plus la part de ces enseignants précaires et souvent à faible expé- 
rience sera forte, ce qui ne pourra que contribuer à rendre difficile l'adaptation des nouveaux étudiants et leur capacité à s'intégrer dans l'enseignement supérieur. (Areser, Quelques diagnostics et remèdes urgents pour une université en péril p. 44)

De nombreux indicateurs pourraient permettre de mesurer l'effet Potemkine. Plus une université est Potemkine, plus le turnover des jeunes maîtres de conférences et des professeurs y est fort,| plus augmente le pourcentage des enseignants non-résidents ou affiliés à des laboratoires d'UM, plus augmente aussi le pourcentage d'étudiants (spécialement chez les titulaires de mention) qui entreprend de migrer vers les UM après le DEUG et, plus encore, après la licence ou la maîtrise. (Areser, Quelques diagnostics et remèdes urgents pour une université en péril p. 88)

Cela vaut aussi pour l'anglais (où il n'y pas non plus de critère syntaxique solide) - à moins, bien sûr, qu'il n'y ait une conjonction de coordination entre deux sous-séquences en $\mathrm{A}$ ou en $\mathrm{B}$.

But the more evidence they uncover and the more they discover about Skinner's private life, | the worse things look for Skinner. (www.anu.co.uk/)

In other words, the worse your project is in technical terms, $\mid$ the lower their initial price quote will be and the more money they will charge you. (www.sauer-int.com)

Allaire et Savelli commentent toutes les deux un petit texte écrit par Obaldia où on trouve des siamoises en cascade :

"Plus je regarde cette photographie d'Obaldia, plus je m'examine, moi, Obaldia, plus je me scrute, plus je me déchiffre, plus je me découvre, plus je me dévisage, plus je m'épie, plus je me provoque, plus je me surprends, plus je me mets à la question, plus je me considère, plus je me reconsidère, | et plus je me trouve un air de famille (R. de Obaldia, Miroirs auto-portraits, in Allaire 1982: 378 et Savelli 1995: 75)

Elles s'accordent à penser que pour des raisons pragmatiques, on ne trouverait guère d'énoncés avec autant de séquences introduites par le comparatif négatif moins. Il est vrai que les constructions en plusplus sont beaucoup plus fréquentes que celles contenant le comparatif moins. Mais est-ce une raison suffisante pour rejeter d'emblée l'hypothèse d'un grand nombre de moins en tête de séquence ? Le petit 
jeu auquel s'est livré l'auteur avec plus serait-il absolument exclu avec moins ? Ne pourrait-on pas imaginer un texte négatif de la même veine - moyennant une petite modification?

\footnotetext{
? "Moins je regarde cette photographie d'Obaldia, moins je m'examine, moi, Obaldia, moins je me scrute, moins je me déchiffre, moins je me découvre, moins je me dévisage, moins je m’épie, moins je me provoque, moins je me surprends, moins je me mets à la question, moins je me considère, moins je me reconsidère, | et [ mieux je me porte "]?
}

Pour conclure, il est donc possible, à mon avis, de ranger les constructions siamoises en deux grands groupes : constructions binaires simples et constructions binaires complexes, en ayant recours à différents critères (d'ordre syntaxique, sémantique, pragmatique et, en langue parlée, intonatif). Les constructions complexes à trois, quatre ou cinq séquences se rencontrent surtout à l'écrit, ce qui explique que l'intonation ne puisse jouer un rôle décisif comme critère dans la détermination de la césure, en français, ni en anglais non plus.

\section{Coordination ou subordination}

Thiersch, qui s'est penché sur la construction anglaise dans un article intitulé The harder they come...!!!, commence par cette remarque judicieuse : "The more one thinks about these constructions, the odder they seem » (1982: 47). Les constructions siamoises ne se distinguent pas seulement des autres constructions corrélatives, mais également d'autres types de constructions. S'agit-il, en français, d'une relation de coordination ou de subordination ? Si la question se révèle épineuse, c'est qu'il n'y a pas, à première vue, de critère syntaxique formel. On dirait qu'il s'agit d'une juxtaposition ou d'une coordination asyndétique de deux propositions principales. Pourtant, la focalisation sur les deux comparatifs déplacés à gauche laisse quand même penser qu'il s'agit d'une construction à part. On constate tout d'abord l'existence d'une relation solidaire entre les deux séquences dont aucune ne peut être omise sans que la phrase ne devienne boiteuse. Si l'on trouve, malgré cette solidarité, des énoncés tels que :

Moins je le vois.... !

Plus ça change...... ! 
c'est que le locuteur ne finit pas sa phrase. Si le locuteur peut se permettre de supprimer la dernière séquence, c'est qu'il s'agit de constructions siamoises devenues de vrais clichés. Normalement, c'est la séquence $B$, c'est-à-dire la conclusion, qui est sous-entendue, comme dans les énoncés cités ci-dessus, mais il arrive aussi que ce soit la séquence A qui est sous-entendue. Savelli (1993: 173) mentionne à propos de ces ellipses le titre d'un film américain, traduit en français de la façon suivante:

Plus dure sera la chute.

The harder they fall. (le titre américain)

Elle en propose différentes interprétations : comparatif la chute sera plus dure (que ce que tu l'imagines) ou la chute sera d'autant plus dure (que..) ou segment autonome de type conclusif à la suite d'une argumentation d'autant plus dure sera la chute, mais elle finit par conclure que ce n'est pas une construction siamoise puisqu'il s'agit visiblement d'une conclusion. Et si pour une fois c'était la séquence A qui avait été sous-entendue et la conclusion qui avait été retenue ? Dans ce cas, nous aurions, dans l'exemple en question, une conclusion exprimée par la séquence B. Plus loin dans sa thèse, Savelli mentionne pourtant un exemple qui semble étayer la validité de cette hypothèse.

Les choses vont toujours vers l'extrême opposé. Plus on monte haut plus dure sera la chute. Je suis prêt à me briser les os en tombant. (in Savelli 1993 : 174)

La structure complète du titre anglais cité plus haut prouve le bien-fondé de cette idée :

The harder they come, the harder they fall. (Jimmy Cliff 1972)

C’est donc bien la séquence A qui a été sous-entendue dans ce titre. Normalement, les deux séquences sont si étroitement liées qu'aucune d'elles ne peut être sous-entendue. L'omission d'une séquence est seulement possible quand le locuteur est sûr de se faire comprendre, par exemple quand il s'agit d'énoncés proverbiaux ou de clichés. Et ce titre où c'est la première séquence qui a été omise est devenu un cliché éculé. Il apparaît souvent dans la langue courante - parfois un peu déguisé:

Après la dissolution ratée. Le référendum perdu. Chirac : plus dure sera la fin. (Le Nouvel Observateur, 28 juin 2005) 
Plus dure sera la sortie de crise, prédit Jean Peyrelevade. (www.lepoint.fr/actualites-economie)

Les deux séquences de la construction siamoise sont donc solidaires et inséparables. Reste à déterminer la relation exacte entre les deux séquences. Coordination ou subordination ? Subordination syntaxique ou subordination sémantique ? La réponse n’est pas évidente pour deux raisons. Il n'y a pas dans toutes les langues de subordonnant syntaxique et - ce qui complique les choses - en français, la conjonction et apparaît souvent dans la césure entre les deux séquences.

\subsection{Subordonnant}

En français standard, une proposition subordonnée est toujours introduite par un subordonnant - selon la grammaire normative. Comme la séquence A n'est pas introduite par un subordonnant, on devrait donc logiquement en conclure qu'il ne s'agit pas d'une subordonnée. Cette conclusion ne semble cependant pas satisfaisante. Peut-on parler de subordination, s'il n'y a pas de subordonnant ? Cela est tout à fait possible en danois et en anglais où les propositions subordonnées non introduites ne sont pas rares du tout. Dans les trois autres langues, au contraire, les propositions subordonnées sont presque toujours introduites par un subordonnant. Sandfeld (1965: 451) ne se prononce pas clairement sur la relation entre les deux séquences de la construction siamoise. Après avoir parlé des constructions avec d'autant plus ou d'autant moins suivies d'une comparative, il dit : «Un autre procédé consiste dans la juxtaposition (c'est moi qui souligne) de deux propositions introduites par plus ou moins » et il continue en précisant : « Dans ce cas, c'est la première des propositions qui correspond à la subordonnée des cas précédents ». Selon Allaire (1982: 342), il ne faut pas confondre le plan syntaxique et le plan sémantique. Si l'on mélange les deux niveaux, « il devient radicalement impossible de définir les phrases en plus.....plus (..) ». A son avis, les constructions siamoises contiennent bien une proposition qui est sémantiquement dépendante de l'autre. Mais comme cette proposition n'est pas signalée par un « subordonnant » et qu'elle « se trouve coordonnée à sa voisine, comme le souligne l'apparition de la conjonction et au centre du système ", il ne s'agirait pas selon elle d'une relation de subordination sur le plan syntaxique, mais d'une relation de coordination. 
Il existe pourtant, en français aussi, certains types de juxtaposition de propositions apparemment indépendantes dont les linguistes s'accordent cependant à penser que l'une est subordonnée par rapport à l'autre. C'est notamment le cas de deux propositions au conditionnel (passé) dont la première est considérée comme une conditionnelle ( $s i$ on avait eu...).

Le pouvoir n'a pas tenu ses engagements. On ne pensait pas que l'affrontement avec les forces de l'ordre prendrait cette tournure. Et puis il lâche : « on aurait eu les camions, la situation aurait été différente. (Le Nouvel Observateur 28- novembre 2002 p. 30)

Dans des énoncés non introduits, l'intonation, les relations sémantiques et le contexte jouent un grand rôle pour le décodage. Est-il vraiment nécessaire distinguer de façon tranchée entre subordination syntaxique et subordination sémantique9. Je ne le pense pas.

Dans The Philosophy of Grammar (1934: 251), Jespersen dit à propos de l'anglais, qui n'a pas de subordonnant non plus: «In ordinary E. the two members have exactly the same construction, and there is nothing to show which is the dependant and which the principal clause in «the more he gets, the more he wants»; but in Dan. and in G. (and formerly also in E.) the word order in such cases shows that the first is the determinant and the second the determined. ». L'exemple suivant illustre bien ce que dit Jespersen à propos de l'ancien anglais (voir la parenthèse de Jespersen).

Thus, the dog behind could never catch up with the one in front. The faster he ran,| the faster ran the one he was after, and the faster ran all the dogs. . (www.pagebypagebooks.com)

Plus tard, dans A Modern English Grammar (1940/52: 380) Jespersen dit à propos de l'introducteur «The usual expression is the...the... with a comparative after each the; the is $\mathrm{OE}$ instrumental by to denote the difference. Complete proportionality is generally out of the question. The conditioning clause is generally placed first; very often the copula is is left out». Jespersen precise un peu plus loin (381) que l'inversion est assez courante dans l'énoncé principal postposé: «Inversion is pretty frequent in the postposed main statement, especially if it is pre-

9 Voir Gadet 1992:85 à propos du mythe de la simplicité. 
ceded by more than one the-clause; this often contributes to clearness as to which is the superordinate». Et de citer un exemple emprunté à Jane Austen:

The more I know of the world, the more am I convinced.

L'ordre le plus courant en anglais moderne est l'ordre direct, mais on trouve parfois l'inversion du sujet :

Minnie thinks perhaps everything will become clearer in due course, so she reads on, but the deeper she gets into the story the thicker is the fog of her incomprehension (David Lodge, Author, Author p. 20)

Selon Quirk and Greenbaum (1979: 756), «the initial proportional clause is regarded as an adverbial within the main clause. Since both clauses in a proportional sentence are of the same general pattern, however, it is not obvious why the first part of the sentence, and not the second, should be treated as the subordinate clause. Apart from the parallel with as ...(so) sentences, the reason for this analysis lies in the general principle that subordination by means of correlative conjunctions [...] entails placing the subordinate clause first. There is also a similarity between the first part and an if-clause ». Leur point de vue peut être illustré par l'énoncé suivant qui montre que la séquence A est parfois à interpréter comme une conditionnelle :

\begin{abstract}
What's at stake? The more students read, the more they know. The more they know, the smarter they become. The smarter they become, the longer they stay in school. The longer they stay in school, the more job and career options they will have later on, and the more money they are likely to earn. The more they earn and the more they like their jobs, the happier and healthier they will be. And yet, Trelease estimates that 90 percent of fifth-graders devote less than 1 percent of their free time to reading, while spending 33 percent of their free time watching television. (rbsms.pasco.k12.fl.us/Stewart/read)
\end{abstract}

Pour l'anglais, Culicover \& Jackendorf (1999) et Taylor (2004) ont signalé d'autres indices de subordination : les 'questions-tag' et la possibilité d'avoir le subjonctif dans la séquence B. Aux questions-tag en anglais (isn't he/she/it - did he/she/it) correspond l'expression n'estce pas en français. En anglais, la question-tag 's'accorde' avec le sujet de la séquence B (matrix clause). Selon Taylor (2004): « Clause $_{1}$ and clause $_{2}$ behave like a subordinate and a matrix clause, respectively [in] 
The more pizza Romeo eats, the more disappointed Juliet is, isn't she.

*The more disappointed Juliet is, the more pizza Romeo eats, isn't she.

If Romeo eats more pizza, (then) Juliet is more disappointed, isn't she?

*If Juliet is more disappointed, (then) Romeo eats more pizza, isn't she? »

Les exemples non précédés d'un astérisque sont censés montrer que la séquence A du premier exemple est bien une subordonnée. Malheureusement, la question-tag n'est pas de la même utilité en français pour prouver la subordination syntaxique de la séquence $\mathrm{A}$, étant donné que l'expression est complètement figée et n'existe que sous cette forme. Tout ce que la question-tag n'est-ce pas peut démontrer, c'est la solidarité entre les deux séquences de la construction siamoise. Pour l'anglais Culicover/Jackendoff (1999: 548) et Taylor (2004: 2) relèvent un autre indice : l'emploi du subjonctif dans la séquence B :

a. I demand that the more John eats the more he pay(s)

b. *I demand that the more John eat, the more he pay(s)

Selon Culicover \& Jackendoff, «subjunctive morphology may appear on $\mathrm{C}_{2},[\ldots]$ The subjunctive may not appear on $\mathrm{C}_{1}$ ». Taylor constate: Clause ${ }_{2}$ can host subjunctive case, but clause ${ }_{1}$ cannot. » Il ne m'a pas été possible de trouver d'exemples authentiques avec le subjonctif dans $\mathrm{C}_{2}$ après le verbe demand ou d'autres expressions volitives en anglais. Le seul exemple sur Google avec un subjonctif en $\mathrm{C}_{2}$ c'est l'exemple construit par Culicover et Jackendoff et cité par Taylor. Je rappelle qu'en anglais, le subjonctif n'est pas obligatoire après les expressions volitives.

En français, on trouve souvent des siamoises enchâssées dans des complétives après des verbes déclaratifs, mais il est plutôt rare de trouver des siamoises après des expressions volitives. C'est uniquement après le verbe falloir que j'ai pu relever quelques exemples - la plupart avec des formes verbales qui ne permettent pas de distinguer entre le présent de l'indicatif et le présent du subjonctif. Mais voici quelques exemples avec un subjonctif identifiable :

Comme le but des prélèvements obligatoires n’est pas seulement de financer l'action de l'Etat, mais de réduire les inégalités, il faut que 
plus le revenu augmente, plus la part d'impôt payé soit forte. La seconde est le versement de revenus indépendants de l'activité productive. (www.academon.fr/Dissertation)

En l'absence de fondations et pour qu'un édifice soit stable, il faut que plus la construction est haute, plus sa base soit large (ainsi la pyramide est la stable des constructions) (www.guidecasa.com)

L'emploi du subjonctif dans la séquence $B$ et non dans la séquence $A$ semble prouver que la séquence A est bien une sorte de subordonnée puisqu'on ne trouverait jamais le subjonctif régi par un verbe volitif dans des subordonnées qui se mettent normalement à l'indicatif (introduites par quand par exemple). J'ai relevé un seul contre-exemple avec le subjonctif dans les deux séquences après il semble que, donc même dans la séquence A. L'exemple avec le subjonctif en A est cependant contre-intuitif.

Il semble aussi que plus on soit âgé, plus on utilise sa monnaie pour payer. (www.desjardins.com)

Il semble que plus le temps passe, plus il soit difficile pour un prestataire de réintégrer le marché de l'emploi. (jobboom.v1.myvirtualpaper. com/magazine)

Si on se tourne vers les exemples en allemand, danois, espagnol et italien, on verra que dans ces langues, le rapport de subordination est marqué de différentes façons. En allemand et en danois, qui sont des langues V 2, l'influence de l' introducteur + le comparatif déplacé à gauche n'est pas la même dans les deux séquences. La séquence A observe, dans les deux langues, l'ordre normal des subordonnées : l'ordre SV en danois et en allemand le verbe est placé en dernière position. Dans la séquence B, par contre, le détachement de l'introducteur et du comparatif provoque l'inversion du sujet, ce qui prouve que cette séquence est une proposition principale. Pour ces deux langues, la subordination syntaxique entre $\mathrm{A}$ et $\mathrm{B}$ est donc bien solide.

Je mehr Sie über Ihr Produkt wissen, um so besser können Sie es verkaufen. (www.lernklick.ch/bewerbungsregeln)

Jo højere man kommer op i magtpyramiden, desto stærkere bliver magtkampen - og så holder kvinderne op (www.nykredit.dk/) 
En espagnol, la séquence A est toujours introduite par le relatif cuanto et en italien, elle peut être introduite par le relatif quanto et quand elle ne l'est pas, on pourra toujours l'insérer. Pour ces deux langues, la relation de subordination est soit clairement établie soit facile à établir.

Cuanto más rápido vayas, más alta será tu puntuación. (www.expocursos.com)

Quanto più le cose appariranno incerte, tanto più mi sentirò sicuro perché l'incertezza è la via alla libertà. (www.tqs.it/nautilus/chopra)

\subsection{Cataphore}

$\mathrm{Si}$, comme nous venons de le voir, la subordination syntaxique n'est pas clairement marquée, ni en français ni en anglais, l'emploi du pronom cataphorique dans la construction siamoise indique pourtant que, dans ces deux langues, la relation de subordination syntaxique peut être marquée faiblement. Le pronom cataphorique, qui ne s'emploie jamais dans les coordinations, est assez fréquent dans les subordonnées.

*Il a pris son chapeau et Pierre est parti.

Quand il était petit dans le « quartier pauvre de Neuilly », selon l'expression d'un ineffable « vieil ami » rapportée par le Nouvel Observateur, Nicolas Sarkozy avait une hantise : que ses copains d'enfance découvrent dans le réfrigérateur familial ces vulgaires tranches de saumon fumé sous cellophane, quand le leur venait des meilleurs traiteurs de l'Ouest parisien. (www.humanite.fr/2007-12-31_Politique)

Comme il l'a fait durant cette campagne, Nicolas Sarkozy est venu sur le terrain des valeurs, car pour lui, « ce fut une campagne aux prises avec une crise morale »( m.marianne2.fr)

S'il voulait vraiment faire qq chose, le gouvernement annulerait son gros paquet fiscal à l'usage des gens qui ne sont pas dans le besoin ! (www.rue89.com)

Le fait que l'on trouve également le pronom cataphorique dans les constructions siamoises peut donc être considéré comme un indice syntaxique de la relation de subordination qui existent entre les deux séquences $\mathrm{A}+\mathrm{B}$. 
Plus elles sont énormes, moins elles ont de fondement, | plus les rumeurs sont assurées d'une belle carrière en Orient. (Frantext, Grèce Michel de / La Nuit du sérail 1982, 326 )

Plus ils sont diplômés, plus les pères aident les petits.. (ecolesdifferentes.free.fr)

Mais, plus ils sont âgés, plus les Franciliens connaissent de fortes difficultés à l'écrit. (www.insee.fr/fr/insee)

Plus elle dure, plus la déception est grande. (www.lmda.net)

Plus il est infusé, plus le thé est fort. (www.linternaute.com)

Camescopes : plus il est cher, moins le zoom est puissant (fr.answers. yahoo.com)

Différentes études internationales et françaises montrent que plus son niveau académique est élevé, plus l'enseignant est à l'aise dans sa discipline et meilleure est la réussite de ses élèves, y compris en ZEP (Le Nouvel Observateur 5-11 février 2009 p. 43)

Thiersch (1982: 51) a fait la même observation en ce qui concerne l'anglais : " Another argument can be found by checking pronominalization. In general, anaphoric reference is allowed backwards into subordinate clauses, but not into coordinate structures:

$\mathrm{John}_{\mathrm{i}}$ went to the store and he bought some apples.

${ }^{*} \mathrm{He}_{\mathrm{i}}$ went to the store and John $n_{\mathrm{i}}$ bought some apples.

If he goes to the store, John ${ }_{\mathrm{i}}$ will buy some apples.

Similarly:

The more John ${ }_{\mathrm{i}}$ ate, the fatter he $\mathrm{i}_{\mathrm{i}}$ got.

The more he ${ }_{i}$ ate, the fatter John ${ }_{i}$ got.»

Plus tard, McCawley (1988: 177) a compté cet emploi du pronom cataphorique en anglais comme une propriété « inherited from conditional constructions ». Comme il a été montré ci-dessus, c'est une propriété qui est commune aux subordonnées. La simple possibilité d'employer le pronom cataphorique indique qu'il s'agit aussi en français et en anglais de subordination syntaxique. 


\subsection{Emploi de la conjonction et dans la césure}

Comment interpréter l'emploi de la conjonction et dans les siamoises en français ? Les conjonctions et et donc sont très fréquents devant la construction entière. Ici, il sera uniquement question du et qui peut apparaître entre les deux séquences. Pour certains, l'apparition de la conjonction entre A et B serait un argument fort en faveur d'une relation de coordination entre A et B. Mais est-ce vraiment l'emploi coordinatif de et que l'on trouve entre les deux séquences? A mon avis, il faudrait distinguer deux emplois de la conjonction dans les constructions siamoises : un emploi coordinatif et un emploi conclusif. Peut-on prouver le bien-fondé d'une telle distinction ? Comme le montrent les exemples suivants, la conjonction et peut, dans son emploi coordinatif entre les sous-séquences, alterner avec la conjonction ou :

\footnotetext{
Plus l'étoile est fine, ou plus son intensité au centre est importante, | meilleure est la focalisation. (www.astrosurf.com)

En effet, plus l'écart entre le coût du travail d'un salarié jeune et d'un salarié senior est important, | et moins le salarié âgé est attractif à l'embauche, ou plus son maintien dans l'entreprise se révèle coûteux. (www.cadremploi.fr/emploi)
}

La conjonction ou apparaît uniquement entre des sous-séquences, elle est exclue dans la césure entre A et B. En effet, employer la conjonction ou dans la césure serait nier d'emblée la corrélation qu'on essaie justement d'établir entre deux variations simultanées (augmentation et/ ou diminution).

Dans la césure entre les deux séquences, on peut mettre soit une virgule soit la conjonction et, qui n'a pas ici sa fonction première de coordination. L'emploi du et conclusif est un phénomène ancien. En témoignent les exemples cités par Antoine (1958: 871) :

Plus y labeure, et plus me plaist. (Froissart, XIV,3)

Plus il estoit embrouillé, et plus il s'embrouiloit. (Commines, IV,1)

Selon Allaire, la fonction de et serait de coordonner des coordonnants : "Son rôle n'est autre que de coordonner des coordonnants; il entre donc lui aussi dans la série additive, potentiellement infinie, de la phrase » (1982: 366). Les coordonnants dont parle Allaire sont les comparatifs en position initiale : « la grammaticalisation de la position adverbiale est indissociable de l'application du principe de réitération. C’est le 
déplacement de l'adverbe qui le rend coordonnant » (1982: 364). Il est déjà un peu difficile de considérer les comparatifs initiaux comme des coordonnants. Comment coordonner, de surcroît, ces coordonnants ? Normalement, les coordonnants ne se combinent pas entre eux.

Savelli (1993: 276) parle de trois positions possibles de la conjonction et : antéposition, position axiale et position finale. L'antéposition comprend les cas où la conjonction et précède la construction entière. Les deux autres positions comprennent, toutes les deux, à en juger d'après les exemples cités, l'emploi de la conjonction et dans la césure. La conjonction et occupe la position axiale dans une structure simple et la position finale dans une structure complexe. La conjonction apparaît dans les deux cas juste avant la séquence $B$. Comme je distingue entre séquence et sous-séquences, la position axiale et la position finale sont, de mon point de vue, la même position : la césure. Savelli parle à propos de quelques exemples de la valeur conclusive de et. C'est une piste qu'il faudrait explorer. Certains exemples de mon corpus montrent qu'en dehors de la conjonction et, on trouve également les connecteurs conclusifs donc et par conséquent dans la césure, comme le montrent les exemples mentionnés ci-dessous. L'emploi de ces connecteurs étaye l'hypothèse de la valeur conclusive de et dans la césure.

Cette force, c'est le poids de l'eau qui se trouve au dessus de votre main. Or, plus vous descendez profond, plus le poids de l'eau au dessus de vous est important, | donc en fait, plus la pression est importante. (scio.free.fr/mecaflu/archimede.)

Plus les individus sont semblables, plus ils s'imitent let donc plus ils se ressemblent. ...(www.polytechnique.fr/)

Notre hypothèse est que plus les enfants sont grands, moins ils ont de représentations incorrectes | donc plus ils ont de stéréotypes. (tecfa. unige.ch/etu/)

En général, plus l'objectif est grand, plus il y a de lumière recueillie | et par conséquent, plus l'image est lumineuse. (www.sisl.ch)

En italien, la conjonction $e$ apparaît aussi dans la césure entre les deux séquences aussi bien dans la langue courante que dans la langue littéraire.

E piú sforzi fa per liberarsi | e piú si fa affondare i denti della tagliola dentro la carne. (Moravia in Spore 1975: 37) 


\begin{abstract}
...sai, sono cambiata, ora sto meglio? diceva lei a lui, senza confessare il fatto banale che più si va avanti | e più le cose si complicano. ...(www.bresciaweb.com/)

Cosa pensi del cinema italiano contemporaneo? Io credo questo: più le cose sono cupe e drammatiche | e più offrono una via all'umorismo. (www.santarcangelofestival.com)
\end{abstract}

Dans les autres langues, la conjonction de coordination s'emploie exclusivement entre les séquences partielles $\left(A_{1}, A_{2}\right.$, etc.) et avec sa fonction première de coordination.

Pour conclure, on peut donc constater que la comparaison entre plusieurs langues montre que la séquence A est subordonnée à la séquence B en allemand, danois, espagnol et souvent aussi en italien et que, même en français et en anglais, il existe la possibilité d'un marquage syntaxique faible de la subordination (l'emploi du pronom cataphorique, l'emploi du subjonctif en B) De plus, on peut constater que dans la césure, la conjonction et en français n'a pas pour fonction de coordonner les séquences $\mathrm{A}$ et $\mathrm{B}$, mais de souligner la conclusion.

En ce qui concerne l'organisation syntaxique, on peut constater que dans les six langues examinées, les constructions siamoises se placent différemment sur l'échelle de Lehmann : « hierarchical downgrading »(1988 :189). Dans le cas du français et de l'anglais, il n'est question que d'une subordination syntaxique faible, souvent inexprimée. A l'opposé se trouvent l'allemand, le danois et l'espagnol, où il est question de subordination syntaxique forte, l'italien se situant entre ces deux pôles.

\title{
6. Syntagmes comparatifs continus ou discontinus
}

Comme il a été constaté plus haut, la caractéristique de la construction siamoise est la focalisation sur deux comparatifs de suite. Le déplacement à gauche d'un syntagme comparatif dans les deux séquences $\mathrm{A}+\mathrm{B}$ a été grammaticalisé dans les six langues. Le comparatif peut être déplacé vers la gauche soit en entier soit en partie seulement. Selon Allaire (1982: 355) c'est seulement la position de l'adverbe (plus/moi$n s)$ qui a été grammaticalisée : «Ainsi grammaticalisée, la position de l'adverbe contribue à la définition du système ... » et plus loin, elle précise « Or nous venons de rappeler que l'adverbe doit à sa fonction 
syntaxique d'occuper la première place dans chacun des deux membres du système dont il assure la cohésion textuelle » (1982: 403). En limitant la grammaticalisation au seul adverbe, Allaire exclut, de fait, les comparatifs synthétiques (meilleur, moindre, pire, mieux). Mieux et meilleur sont pourtant très fréquents dans la langue courante dans cette construction tandis que moindre et pire apparaissent plutôt dans le langage technique.

Plus l'assise est large et profonde, meilleure est la répartition de l'effort de butée sur le sol et moindres sont les déplacements. (www.ressource.fr)

Plus l'effort est excessif, pires sont les symptômes et moins l'effort est excessif plus les symptômes sont légers. ...(Www.agoraphobie. com)

Plus un devis est détaillé et complet, moindres sont les éventuelles déconvenues du commanditaire. Exemple de devis. (www.audiovisuel-til.net)

Plus les années passent, pires sont les scénarios d'épouvante qu'on nous sert sur l'éventualité d'un OUI à la souveraineté. (www.vigile. net/pol/)

Et il semble que plus on a commencé à fumer jeune, pires sont les manifestations.(www.cybersciences.com)

Il est clair que c'est la position du comparatif qui a été grammaticalisée, qu'il s'agisse d'un comparatif synthétique ou d'un comparatif analytique. Il suffit de regarder tous les exemples avec des comparatifs synthétiques et analytiques - en tête de séquence en français et dans les 5 autres langues. Dans le cas des comparatifs analytiques, il est possible de ne déplacer à gauche que l'adverbe comparatif, c'est pourquoi il faudrait distinguer entre 'syntagmes comparatifs continus' et 'syntagmes comparatifs discontinus. En étudiant les adjectifs 'nés' ou les vrais adjectifs, on voit qu'en français, espagnol et italien, le comparatif analytique est plus fréquent. Dans les langues germaniques, c'est l'inverse. Ici les comparatifs synthétiques dominent.

Dans les syntagmes discontinus, les constituants d'un comparatif analytique sont séparés l'un de l'autre : l'adverbe comparatif est déplacé vers la gauche, en position initiale, alors que l'adjectif comparé gar- 
de la position que lui donne sa fonction grammaticale. Comme, dans les langues romanes, les comparatifs analytiques sont plus nombreux que les comparatifs synthétiques, les syntagmes discontinus y sont également très fréquents. Théoriquement, les comparatifs synthétiques se prêtent uniquement à un déplacement à gauche du syntagme comparatif 'en entier', comme il sera ressorti des exemples précédents, mais 'sur le terrain' c'est autre chose. On trouve parfois une sorte de comparatifs discontinus non-autorisés comme le montrent les exemples suivants :

Plus y'en a, plus c'est mieux ! On en voudrait au moins 30, pour les 30 ans de Stonewall ! (perso.wanadoo.fr/)

Mais bon, comme le dit le proverbe, plus c'est crétin plus c'est bien. (www.krinein.com)

Plus ta version sera récente et plus mieux ça sera! (shamo.planet)

En gros, parfois il a envie de vomir, et il vomit, et plus il angoisse et stresse, plus c'est pire. (forum.aufeminin)

Il va sans dire que ces syntagmes comparatifs discontinus, qui relèvent de langue spontanée ou populaire, ne sont pas de mise en français standard.

En français, le comparatif peut être déplacé à gauche s’il remplit la fonction d'attribut (meilleur sera son résultat /plus grand sera son effort...) ou de complément adverbial (plus vite il démarre...), mais contrairement aux langues germaniques, le comparatif ne peut pas être déplacé à gauche s'il remplit la fonction d'épithète dans un syntagme nominal en fonction d'objet. Cette contrainte s'explique par l'impossibilité générale d'antéposer l'objet. En anglais et danois, on peut - sans problemès - antéposer l'objet, comme il ressort des exemples suivants (les syntagmes concernés sont indiqués en italiques):

The fewer people there were living at home the bigger house they got. (www.uoregon.edu)

F.eks. betyder fastfrysningen af ejendomsværdiskatten jo kun noget for dem, der har hus, og jo større hus man har, des større skattelettelser får man. ...(www.marxist.subnet.dk) 
En français, il ne serait donc pas possible de traduire ces énoncés en commençant par un syntagme comparatif continu détaché en position initiale:

*Moins il y avait de personnes à la maison, plus grande maison ils avaient.

*Plus grande maison on a, plus grand allègement d'impôts on aura.

On serait obligé d'employer un syntagme comparatif discontinu :

Plus la maison (que l'on possède) est grande, plus l'allègement d'impôts sera grand.

Plus la maison (que l'on possède est grande, plus grand sera l'allègement.

C'est-à-dire que l'on est obligé d'exprimer la prédication sous-jacente, comme on le voit aussi dans l'exemple suivant :

Plus grand est le secteur qu' elle contrôle, mieux elle est placée pour faire fructifier les capitaux parce qu' elle traite une quantité énorme d' opérations; (Frantext, Lesourd, J.-A., Gérard C. Histoire économique : XIXe et XXe siècles : t. 11968 p. 79)

En français, un comparatif analytique en fonction d'attribut du sujet peut être déplacé à gauche entièrement ou partiellement (syntagme continu ou discontinu). Il faut préciser que le déplacement à gauche d'un syntagme continu est seulement possible si le sujet est un sujet non-clitique. Si le comparatif tout entier est déplacé vers la gauche, le sujet non-clitique est déplacé vers la droite suivant les règles de l'inversion du sujet dans les constructions tripartites (voir Korzen 1992). Par contre, si le sujet de la séquence est un sujet clitique, le recours à un syntagme discontinu est obligatoire et l'ordre direct SV est également obligatoire.

Syntagmes continus :

Le conseiller juridique devra organiser la copropriété en tenant compte de tous ses aspects spécifiques; plus grand sera son effort, plus grands seront les profits du promoteur. (www.notarius.com/)

Ils pensent que plus grand sera le nombre d'amis impliqués ou responsabilisés dans cette activité spécifique, plus importants seront les résultats obtenus. ... (www.amis.monde-diplomatique.fr/) 
Les événements démasquent les hommes : plus grande est la catastrophe et mieux elle nous dénude. (Frantext, Mauriac, F. Journal du temps de l'Occupation / 1944, p.351)

Plus nombreux seront les adultes à décéder du sida, plus nombreux seront les orphelins.(www.un.org/News/fr-press)

\section{Syntagmes discontinus :}

Distance sociale: plus elle est grande entre les interlocuteurs, plus les adoucisseurs sont de mises. (C. Kerbrat-Orecchioni, les actes de langages dans le discours p. 106)

Cadre est un titre, non une fonction. Mieux vaut l'être que ne pas l'être : comme tout le monde passe son temps à faire le travail de la personne du dessus, plus on est haut, moins on a à faire - plus vous êtes important moins vous travaillez, c'est une loi d'airain du monde du travail. (Corinne Maier, Bonjour paresse p. 69)

Plus elle semble rude et impitoyable, plus elle est tendre au fond, et sourcilleuse, et attentive; (Frantext, Dutourd, Jean, Pluche ou l'amour de l'art 1967 p.53)

Il n’y a pas de mystère. Plus les Etats ont privilégié la protection sociale, plus le nombre de détenus est faible (Le Nouvel Observateur 27-03-03 p. 54)

En espagnol et en italien, on trouve aussi des syntagmes continus et discontinus. Il faut se rappeler que dans ces deux langues, le sujet clitique n’est pas exprimé, normalement :

Cuanto más importantes son los medios financieros destinados a la alimentación, más se decantará el individuo por las grasas animales. (www.subneo.net)

Cuanto más están profundos los bordes laterales que se han plegado, tanto menos resultará pequeña la cajita. (www.netpark.or)

El problema es que cuanto más están inmersos en el quehacer cotidiano, menos tienen la posibilidad de ver un panorama más amplio y de reflexionar de manera. (www.uaemex.mx/plin/univ)

Cuanto más estamos despiertos más facilmente olvidamos. Después cuando tenemos tiempo anotamos todo exactamente. (mailbox.univie. ac.at) 
Quanto più veloce è la replicazione virale, quanto più rapida sarà la caduta dei CD4 ed il fallimento del sistema immunitario. (www.lila.it/ aree)

Quanto più sono vecchi, tanto più sono egoisti. (perso.chello.fr)

E i bambini? E' vero che piu' sono asciutti piu' sono felici? (www.artetribale.it)

Si vede che, nelle collisioni tra nuclei di piombo la soppressione è tanto più pronunciata quanto più la collisione è «violenta». (wwwps. lnf.infn.it.)

Avec le comparatif d'un adverbe de temps ou de manière, on trouve tantôt un syntagme continu tantôt un syntagme discontinu en français.

«Plus tôt la Turquie sera prête, mieux ce sera pour l'Europe». (www. ataturquie)

«Plus vite il partira mieux ça vaudra ». Les Occidentaux ont multiplié hier les appels au départ du président yougoslave Slobodan Milosevic. (www.bretagne-online.com)

Il est alors souhaitable d'aborder les différents aspects du syndrome de Turner car plus l'enfant est informée tôt, mieux elle réagit et mieux elle est à même de faire face à ses nouvelles réalités. (www-poleia. lip6.fr)

L'expérience s'acquiert au fil des courses et plus tu commences tôt, plus tu apprends vite. (www.sport-up.fr/version2004)

C'est un des points les plus importants d'une hygiène alimentaire. Plus on mange vite, plus on mange et plus on risque de faire des difficultés digestives. ... (www.nutriconseil.com)

\section{Construction quantitative: syntagme discontinu obligatoire}

Quand le comparatif plus ou moins est employé comme quantifieur, le syntagme discontinu est obligatoire quelle que soit la fonction du syntagme nominal dans lequel il est enchâssé. Le syntagme nominal peut avoir soit la fonction d'attribut du sujet, soit la fonction d'objet. Une telle discontinuité n'est pas inconnue dans d'autres constructions quan- 
titatives, on la trouve par exemple dans les questions introduites par combien :

Mais, pour payer ce résultat, combien a-t-il fallu de pertes, de chagrins, de déchirements. (Frantext Gaulle, Charles de / Mémoires de guerre 1954 p. 261)

Plus on est de fous, plus on rit. (Proverbes 1650)

Moins un être humain possède de mots, plus il frappe dur. (Alexandre Petit, $1+1+1$ p. 49)

La langue, nous l'avons dit plus haut, est à la fois un produit collectif et un bien collectif: aucun individu ne peut la créer ou la maintenir seul, aucun individu ne peut empêcher seul sa création ou la supprimer. Et cette propriété collective définit en partie sa valeur: plus elle a d'utilisateurs et plus elle augmente sa valeur. (Jean-Louis Calvet, Pour une écologie des langues du monde p. 81)

Cette année encore, il semble que les remplacements d'été soient placés sous le signe du «moins on est de fous, plus on bosse». (sudfnac. free.fr/charite)

Plus elle connaîtra de choses, mieux elle pourra s'en sortir. (www.liberation.com/)

Présidentielle française: Plus il y a de candidats, moins il y a de choix. (www.ledevoir.com)

En français et en italien, un syntagme discontinu est de règle quand il s'agit d'un comparatif quantifieur tel que plus elle a d'utilisateurs alors que dans les autres langues, il faut employer un syntagme continu:

italien: più è grande il numero di persone che usa la lingua...

anglais: the more utilizers the language has....

danois: jo flere brugere sproget har...

espagnol: cuanto más usuarios tiene una lengua...

\section{Phrase averbale}

Pour les besoins de l'analyse, il est nécessaire de distinguer entre deux types de phrase averbale : une phrase averbale libre qui n'est soumise à 
aucune contrainte et une phrase averbale liée qui ne peut apparaître que dans un contexte bien précis, à savoir dans la séquence $\mathrm{B}$ après la séquence A ou dans une sous-séquence en A. En allemand, anglais, danois et espagnol, la construction siamoise est très courante dans les phrases averbales que celles-ci soient libres ou liées.

Je eher, je besser. (www2.cddc.vt.edu)

Je später um so besser weil es nun mal kein leichter Eingriff ist. (de. answers.yahoo.com)

The More the Better: but not Taxes! Tax and spend is hardly the recipe for economic success. (www.freema.org/Articles/Taxes)

The more the merrier. (www.bartleby.com)

Jo større, jo bedre - i hvert fald når det gælder innovationsindsatsen. (ing.dk/artikel)

Jo højere kald jo dybere fald. (proverbe)

Han lærte sig ustandselig flere sprog. Jo flere sprog, jo større sammenligningsgrundlag. (Mathilde Walter Clark, Tingenes uorden p. 28)

Cuanto más pobres, más generosos son (Juan Goytisolo, Campos de Nijar p. 92)

Empecemos por lo más básico: una buena mesa y cuanto más grande mejor. (www.ricardsierra.com)

Como dice el refrán "el buen perfume viene en frasco pequeño" por eso invitamos a recordar que si de alcohol se trata, cuanto menos mejor. ... (saludparalavida.sld.cu/)

En français, la construction siamoise s'emploie uniquement dans les phrases à verbe tensé La construction ne se laisse pas réduire en une phrase averbale, comme l'a déjà constaté Claire Blanche-Benveniste (1992: 121): " La forme plus...plus ne peut porter que sur un verbe tensé, avec un sujet réalisé ». Dans sa thèse, Savelli (1993: 205) fait la même observation : " Les verbes doivent tous être tensés - il apparaît, en effet, que ce type d'organisation " siamoise » est obligatoirement marquée dans le temps (présent, passé ou futur) ». Mes recherches 
confirment en partie leurs observations, mais il y a des exceptions. On trouve des siamoises introduites par les quantifieurs plus de ou moins de dans des énoncés figés comme par exemple les proverbes.

Plus de morts, moins d'ennemis. (Dictionnaire des proverbes et dictons $n^{0} 1910$ )

En français moderne, il ne serait guère possible de réduire l'exemple suivant en une phrase averbale, étant donné que plus de peut aussi avoir une signification négative.

Présidentielle française: Plus il y a de candidats, moins il y a de choix. Christian Rioux. (Le samedi 06 avril 2002).

?Plus de candidats, moins de choix.

Comme les apparences sont souvent trompeuses, le lecteur doit donc être sur ses gardes. Dans les exemples suivants, ambigus à première vue, il ne s'agit pas d'une siamoise.

Moins de «schizos», plus de «bouffées délirantes».

Certains psychiatres parlent aujourd’hui de «bouffées délirantes» là où d'autres voient une shizophrénie. Les médicaments sont alors prescrits pour une durée limitée. (www.alterpsy.org/bouffees)

Pourquoi ne pas apprendre à mélanger son fruit et son yaourt? On fait d'une pierre deux coups: moins de sucre, et plus de fruit (Le Nouvel observateur 24-10-02 p. 2002)

Une lecture attentive montre clairement qu'il n'est pas question, dans ces énoncés, d'une construction siamoise. Il n’y a aucune relation de subordination syntaxique ou sémantique. Ce sont tout simplement des énoncés qui résument ou expliquent ce qui est dit dans le passage en question.

En français et en italien, le verbe est donc normalement exprimé dans les siamoises comme le montrent les exemples suivants qui ressemblent pourtant, en ce qui concerne le contenu, aux phrases averbales libres dans les autres langues citées ci-dessus.

Evidemment, plus cela dure mieux c'est, mais je pense qu'ils savaient dès le départ que lorsque que les gens commenceraient à se plaindre, leur objectif ... (linuxfr.org) 
L'Algérie doit à tout prix se préserver de cette nouvelle peste et le plus vite sera le mieux, car c'est l'avenir du travailleur algérien qui est menacé ! ... (fr.altermedia.info)

Più si è veloci e meglio è. (www.ultraleggeri.net)

Anzi: più siamo e meglio è! (www.origami-cdo.it)

Antonio Banderas e` proprio come il vino: piu‘ invecchia e meglio e‘. (www.solecuoreamore.it)

Meno si fa e meglio è, probabilmente. (www.vincenzomollica.rai.it)

La restriction qui frappe les phrases averbales dans ces deux langues s'explique peut-être par le fait qu'il n'y a (le plus souvent) pas de mot introducteur. Dans les autres langues, l'introducteur signale la siamoise et le risque d'une erreur d'interprétation est inexistant. En français et en italien, on trouve seulement les siamoises dans les phrases averbales liées. C'est toujours les verbes copules être et essere qui sont sousentendus dans la séquence $B$.

La vocation est un absolu inconditionnel que toutes les réussites altèrent ; plus grande est la vocation, plus vague sa définition projetée. ... (www.evene.fr/citations)

L'un des paradoxes de la légitimité est que plus les droits des citoyens sont faibles, plus grandes les obligations que doit afficher l'État. (Philippe Simonnot, 39 leçons d'économie contemporaine p. 127)

Plus grande est la masse des biens ou des services produits, plus petit leur coût unitaire, puisque les frais fixes peuvent se répartir sur un plus grand nombre de biens ou de services. (Philippe Simonnot, 39 leçons d'économie contemporaine p. 124)

Plus riche est le contribuable, plus grande sa surface sociale et financière, et plus notre législation s'évertue à alléger sa charge sociale. (F. Mitterrand, La rose au poing p. 142 in Allaire 1982: 394)

L'assorbimento di ossigeno è la quantità di ossigeno che cuore, polmoni e sangue sono in grado di trasportare dall'aria inalata alle cellule muscolari del corpo. È la misurazione fisiologica della nostra forma. Quanto più ossigeno e quanto più veloce il trasporto, tanto maggiore l'assorbimento di ossigeno. (esercizio.primavi.com) 


\section{Contenu sémantique}

Après avoir analysé la syntaxe des constructions siamoises, il faudrait maintenant étudier le sens de la construction. Comme les constructions contiennent des comparatifs, nous avons indéniablement affaire à une comparaison. Reste à savoir ce qui est comparé et à quoi.

Quand ils évoquent le sens de la construction, bon nombre de linguistes parlent de proportionnalité ou de gradation. Ainsi, Sandfeld (1965: 450) qualifie ces propositions de 'propositions de proportion' et dit que " deux actions ou deux faits augmentent ou diminuent dans la même proportion ». Selon Riegel et alii (1994: 516) " le système exprime soit l'équivalence quantitative [autant-autant] soit la variation proportionnelle [plus-plus] ». Zifonun et alii (1997 III 2337) parlent de « Proportionalsätze » pour l'allemand. Quirk/Greenbaum (1979: 756), parlent de proportional sentences. A. Hansen (1967: II, 275) ne parle pas de proportionnalité pour le danois mais souligne qu'il s'agit d'une gradation interdépendante exprimée par les deux comparatifs. Il a été démontré récemment qu'il ne s’agit pas de proportionnalité (voir par exemple Cornulier (1988), Savelli (1993), Beck (1997) et Abeille/Borsley (2005)), mais de variations 'simultanées' ou parallèles. Le terme covariation utilisé par Abeille et Borsley me semble plus apte à exprimer le phénomène dont il est question puisqu'il signale une corrélation entre les variations dans le temps de deux ou de plusieurs grandeurs (voir TLF).

Beck fait une analyse sémantique des " comparative conditionals " (CC) et entend démontrer comment « the desired semantics is derived from the syntax » (1997: 229).

Pour Beck, la construction siamoise est une construction conditionelle avec une subordonnée et une principale tout comme les constructions conditionnelles ordinaires. Ce qui veut dire que la première séquence fonctionne comme la condition et la dernière comme la conséquence. Après avoir analysé de façon pertinente le rôle des deux comparaisons dans la construction, elle conclut que la comparaison s'établit à l'intérieur de chaque proposition entre différents degrés. Il est question d'une quantification universelle qui porte sur des mondes possibles, des temps ou sur des individus (1997: 237). 
Beck distingue entre use et part of their meaning: «I think that CCs can be used to describe functionally dependent relationships, or also causal relations; perhaps because of their peculiar semantics, they strongly tend to be used in this way, as for instance in $36^{10}$. This might be why many people think (c'est moi qui souligne) that it's part of their meaning ». (1997: 245). Elle précise qu'à son avis, les CC n' expriment pas une relation causale : « But I do not think that anything that expresses a causal relation or a functional dependence of the difference degrees is actually part of the meaning of the CC constructions ».

A mon avis, il faut, pour déterminer le sens d'une siamoise, faire entrer en ligne de compte à la fois la syntaxe, la sémantique et la pragmatique. La solidarité entre les deux comparaisons internes à chaque séquence (les comparaisons dont a parlé Beck) montre bien que pour le locuteur c'est l'ensemble, c'est-à-dire la relation entre les deux, qui est porteur de sens. S'il a mis ensemble ces deux comparaisons, ce n'est pas par hasard. En ce faisant il 'oblige' l'interlocuteur à inférer (selon le principe de pertinence) qu'il y a une relation entre les deux. Parfois le contexte amène l'interlocuteur à interpréter cette relation comme une construction conditionnelle. C'est le cas de l'exemple suivant.

\footnotetext{
What's at stake? The more students read, the more they know. The more they know, the smarter they become. The smarter they become, the longer they stay in school. The longer they stay in school, the more job and career options they will have later on, and the more money they are likely to earn. The more they earn and the more they like their jobs, the happier and healthier they will be. And yet, Trelease estimates that 90 percent of fifth-graders devote less than 1 percent of their free time to reading, while spending 33 percent of their free time watching television. (rbsms.pasco.k12.fl.us/Stewart/read.)
}

D'autres fois l'interlocuteur est amené à y voir une relation de cause à effet grâce à l'inférence courante, banale, non-logique post hoc, ergo propter hoc. La relation causale est de nature dynamique : une variation en A entraîne 'automatiquement' une variation en B selon l'expérience de l'interlocuteur.

Frankly the more I hear about him, the less I like him. (David Lodge, A trilogy, Changing places p. 112)

10 Je größer die Geschwindigkeit, desto länger der Bremsweg. 
Plus un titre sonne «américain», plus il fait jeune et branché. ( $(\mathrm{H}$. Astier, Rapport d'évaluation de la politique en faveur du français $2005: 4)$

On peut argumenter en faveur des deux sens proposés en paraphrasant un énoncé comme Moins je le vois, mieux je me porte de la façon suivante :

a. construction conditionnelle : si je le vois moins, je me porte mieux.

b. relation causale : si je me porte mieux, c'est que (ou parce que) je le vois moins.

Le sens de la contruction se déduit donc conjointement de la syntaxe, de la sémantique et de la pragmatique. Tout dépend du contexte.

Quelle est la différence entre les conditionnelles et causales ordinaires et les constructions siamoises? La comparaison de trois énoncés simples permettra peut-être d’y voir un peu plus clair.

Si je le vois moins, je me porterai mieux.

Si je le voyais moins, je me porterais mieux.

Moins je le vois, mieux je me porte.

Dans les deux premiers exemples, il s'agit d'hypothèses explicites portant sur l'avenir, dans le dernier, par contre, on est dans l'implicite. Cet énoncé se base sur une expérience du passé. La formule plus...plus exprime en condensé cette expérience basée sur un certain nombre d'observations de corrélation entre une séquence $\mathrm{A}$ et une séquence $\mathrm{B}$ où le degré augmente et/ou diminue à chaque fois. Le déplacement à gauche des deux comparatifs permet au locuteur de focaliser sur son expérience en la matière. C'est le contexte qui conduit l'interlocuteur à inférer qu'il s'agit d'une condition ou d'une cause. L'avantage de cette construction est qu'elle laisse un peu dans le flou la signification exacte de l'énoncé comme c'est le cas avec, par exemple, le gérondif (voir Halmøy 1983) et les propositions factuelles en si (voir Stage 1991). Elle permet ainsi au locuteur de se prononcer de manière frappante, nuancée et concise. 


\section{Constructions siamoises normales et constructions inversées}

Dans toutes les langues examinées - à l'exception du français - il est possible d'inverser l'ordre des séquences sans altérer le sens de l'énoncé, quitte à opérer quelques modifications. McCawley (1988: 176) distingue entre normal CCs (siamoises normales) et reversed CCs (siamoises inversées) :

The kinder you are to him, the more he imposes on you (normal CCs).

I understand this problem (*the) less, the more time I spend on it (reversed CCs).

On voit que les reversed CCs de McCawley ne sont pas de vraies siamoises puisque, dans la première partie, il n'y plus d'introducteur et que le comparatif n'est plus déplacé à gauche.

En français, l'ordre A+B est obligatoire. Une inversion des séquences changerait le sens de l'ensemble. Savelli (1995: 68) dit à ce propos: « la permutation des segments est possible, sur le plan morpho-syntaxique, dans le cas de plus ... plus :

Plus il grossit plus il mange

Plus il mange plus il grossit».

En constatant que l'inversion des séquences est possible en français " sur le plan morpho-syntaxique », Savelli laisse entendre qu'une telle inversion est impossible sur le plan logico-sémantique.

Si on inverse l'ordre des séquences tout en gardant le même contenu sémantique dans chaque séquence, on s'aperçoit que l'on a toujours affaire à l'ordre A + B, c'est-à-dire une subordonnée + plus une principale, ce qui veut dire que le sens de l'énoncé change aussi quand on change l'ordre des séquences. Dans tous ces exemples, il est donc toujours question de siamoises normales - les unes, certes, plus vraisemblables que d'autres.

1. Plus tu bois, plus tu as la gueule de bois (AB)

2. Plus tu as la gueule de bois, plus tu bois (AB) 
3. The more you drink, the bigger your hangover (will be) (AB)

4. The bigger your hangover, the more you will drink (AB)

5. Jo mere man drikker, jo flere tømmermænd får man (AB)

6. Jo flere tømmermænd man har, jo mere drikker man (AB)

En anglais et danois, on trouve aussi des constructions inversées (reversed CCs) BA où le comparatif de B est déplacé à la fin de la séquence et où le mot qui fonctionne normalement comme introducteur est omis (the, jo). En danois, l'antéposition de la séquence B entraîne l'ordre normal SV dans la séquence B. Ainsi, correspondant à 3-6, on trouve :

3 bis : Your hangover will be bigger, the more you drink (BA)

4 bis: You will drink more, the bigger your hangover (BA)

5 bis: Man får flere tømmermænd, jo mere man drikker (BA)

6 bis: Man drikker mere, jo flere tømmermænd man har (BA)

Si on commence en anglais par la séquence B, l'introducteur de B est omis - comme le constate Jespersen (1942: 382): « The conditioning clause may be placed last; in that case the comparative with the never begins the main part of the sentence ». En danois, jo + le comparatif est également placé en position finale dans la proposition principale antéposée (voir Hansen 1967: I 50).

This way of behaving is not appreciated by the old players and relationships are getting worse | the more he's getting better. (diazgalerie.free.fr)

\footnotetext{
Most pastimes become more expensive | the more you decide to play and compete. (www.cardcounter.com)

Vi hævdede: "Undersøgelser har vist at folk bliver mere skeptiske over for genteknologi | jo mere de ved.” (www.dias.kvl.dk)
}

L'ordre sujet-verbe dans la deuxième séquence des exemples danois (5 bis et 6 bis) indique clairement qu'il s'agit de la séquence A postposée, l'inversion du sujet étant obligatoire dans la séquence B (la principale) placée après la séquence $\mathrm{A}$, comme le prouve l'exemple suivant où l'on a l'ordre AB. L'inversion est provoquée par l'antéposition de la sé- 
quence A qui fonctionne comme complément circonstanciel dans toute la proposition :

Jo tidligere man starter, jo mere drikker man - også som voksen.

(www.sitecenter.dk/)

(Plus on commence tôt, plus on boit)

En français, la construction inversée n'est pas possible, peut-être parce qu'il n’y a pas d'introducteur spécifique dans cette langue.

1 bis : *Tu as plus la gueule de bois, plus tu bois

2. bis : *Tu bois plus, plus tu as la gueule de bois

En allemand, espagnol et italien, l'ordre B + A est assez fréquent et dans ces cas-là l'introducteur de $\mathrm{B}$ est souvent exprimé, mais pas toujours. L'introducteur normal de B + le comparatif est placé non plus en position initiale mais à la fin de la séquence juste avant l'introducteur de la séquence A. Il est clair que dans une construction inversée, l'introducteur des siamoises ne fonctionnent plus dans B comme introducteur puisque le comparatif n'est plus déplacé à gauche.

Daher kann man sagen: «Wir leben um so mehr, | je tiefer wir glauben.“ (www.st-mariae-himmelfahrt.de)

Dabei ist die Pufferwirkung der Batterie um so besser, je kleiner der Quotient aus Spannungsänderung und Stromänderung ist. (www.wipo. org/pctdb/en)

El peso de los productos no protegidos por una patente es probablemente tanto más pequeño | cuanto más especializado es el establecimiento y cuanto mayor es. (www.alass.org)

La comprensión es mayor |cuanto más amplia sea su red de conocimientos (...) (www.correodelmaestro)

In pratica è tanto più facile non essere colpiti | quanto più velocemente ci si muove. (silmaril.novacomp.it/consigli.htm)

L'accendibilita' e' maggiore | quanto piu' alto e' il numero di cetano. A una elevata accendibilita' corrisponde ... (staff.nt2.it/michele)

Selon Bech (1964: 55) l'antéposition de la séquence B (la proposition principale) serait moins fréquente en allemand. 
Il est à noter que les constructions inversées ne correspondent pas à cent pour cent à la définition de la construction siamoise qui a été donnée au début de l’article puisque dans la séquence B antéposée, le comparatif n'est plus déplacé à gauche. Mais comme il est toujours question de deux comparatifs et que le deuxième comparatif est obligatoirement déplacé à gauche, on peut considérer cette construction non pas comme l'équivalent de la siamoise mais comme une sorte de demi-soeur. Le sens des constructions inversées est proche de celui des siamoises normales, mais elle n’a pas le même tonus.

\section{Tableau synoptique}

\begin{tabular}{|l|c|c|c|c|c|c|}
\hline & Allemand & Anglais & Danois & Espagnol & Français & Italien \\
\hline Structure A+B & + & + & + & + & + & + \\
\hline Introducteur & + & + & + & + & -1 & $(+)$ \\
\hline $\begin{array}{l}\text { Déplacement à } \\
\text { gauche }\end{array}$ & + & + & + & + & + & + \\
\hline $\begin{array}{l}\text { Syntagmes } \\
\text { discontinus } \\
\text { (adjectifs nés et } \\
\text { adverbes) }\end{array}$ & - & - & - & + & + & + \\
\hline $\begin{array}{l}\text { Syntagmes } \\
\text { discontinus } \\
\text { (adj. dérivés) }\end{array}$ & + & + & + & + & + & + \\
\hline $\begin{array}{l}\text { Syntagmes } \\
\text { dicontinus } \\
\text { (quantifieurs) }\end{array}$ & - & - & - & $(-)^{2}$ & + & - \\
\hline $\begin{array}{l}\text { Subordination } \\
\text { syntaxique }\end{array}$ & + & $(-)^{3}$ & + & + & $(-)^{3}$ & +()$^{4}$ \\
\hline $\begin{array}{l}\text { Inversion } \\
\text { syntaxique }\end{array}$ & + & - & + & - & - & - \\
\hline $\begin{array}{l}\text { Inversion } \\
\text { stylistique }\end{array}$ & - & + & - & + & + & + \\
\hline $\begin{array}{l}\text { Conjonction } \\
\text { conclusive' } \\
\text { dans la césure }\end{array}$ & - & - & - & - & + & + \\
\hline $\begin{array}{l}\text { Emploi de la CS } \\
\text { dans une phrase } \\
\text { averbale libre }\end{array}$ & + & + & + & + & - & + \\
\hline $\begin{array}{l}\text { Emploi de la CS } \\
\text { dans une phrase } \\
\text { averbale liée }\end{array}$ & + & + & + & + & + & + \\
\hline
\end{tabular}

Tableau 2. Tableau synoptique 
Une parenthèse autour de +/- indique que quelques exceptions à la règle générale ont été relevées.

1. En français moderne, il n'y pas d'introducteur dans la plupart des exemples relevés.

2. Après más de/menos de on trouve parfois des syntagmes discontinus (más hay de...)

3. Subordination syntaxique faible : référence cataphorique

4. Il y a subordination syntaxique en italien, quand l'introducteur quanto est utilisé dans la séquence $\mathrm{A}$.

5. Par inversion syntaxique il faut comprendre que l'inversion est déclenchée par la fonction syntaxique de la séquence $\mathrm{A}=$ adverbial) dans la séquence B. C'est le cas de l'allemand et du danois qui appartiennent aux langues V2.

6. L'inversion stylistique est facultative.

\section{Conclusion}

Cette étude contrastive de 6 langues européennes aura contribué, du moins je l'espère, à mettre en évidence la particularité de la construction en général et de mieux saisir celle du français. Il est frappant de voir que les ressemblances et les différences ne se laissent pas toujours expliquer par l'appartenance ou la non-appartenance à une même famille linguistique. Les deux parties de la construction sont indissociables. On ne peut supprimer l'une sans supprimer le tout - sauf dans quelques cas exceptionnels. Il est aussi frappant que cette interdépendance, ou plus exactement cette subordination de l'une à l'autre n'est pas toujours signalée dans la forme en français et en anglais. Le fait que les deux séquences soient presque identiques dans leur présentation formelle cache en réalité une relation de subordination comme dans les autres langues examinées. Cette analyse aura montré qu'il n'est pas toujours possible de distinguer nettement entre subordination syntaxique et subordination sémantique. J'espère avoir contribué à élucider la syntaxe, la sémantique et la pragmatique des constructions siamoises. Mais tous les secrets de la construction n’ont certainement pas été dévoilés. Plus on creuse, plus on trouve! 


\section{Bibliographie}

Abeille, Anne/Borsley, Robert 2005: La syntaxe des comparatives en anglais et en français. Colloque Subordination-Coordination [online]. www.cavi.univ-paris3.fr/ ilpga/colloque-coord-subord-2005/pre-textes/Abeille-borsley.pdf.

Allaire, Susanne 1982: Le modèle syntaxique des systèmes corrélatifs. Etude en français moderne.

Université de Lille. Lille.

Antoine, Gérald 1959/62: La coordination en français. Paris: Editions d'Artrey.

Bech, Gunnar 1964: The German Je-clauses. In Lingua 13, 49-61. Amsterdam: NorthHolland Publishing CO.

Beck, Sigrid 1997: On the Semantics of Comparative Conditionals. In Linguistics and Philosophy 20, 229-271.

Borsley, Robert 2004: An Approach to English Comparative Correlatives. Proceedings of the HPSG04 Conference [online]. csli-publications.stanford.edu.

Butt, John/Benjamin, Carmen 1998: A New Reference Grammar of Modern Spanish. London: Arnold.

Cappeau, Paul/Savelli, Marie-Josée 1995: Corrélation ne vaut pas comparaison. In Faits de langue $\mathrm{n}^{\circ} 5$.

Carter, Ronald \& Michael McCarthy 1997: Cambridge Grammar of English. Cambridge : Cambridge University Press.

Cornulier, Benoît 1988: Plus on est chauve, plus on est intelligent. Hommage à la mémoire de Jean Stéfanini. Recueil d'études rassemblées par Claire Blanche-Benveniste, Andre Chervel et Maurice Gross. Université de Provence.

Culicover, Peter W./Jackendoff, Ray 1999: The View from the Periphery: The English Comparative correlative. In Linguistic Inquiry, volume 30, number 4, Fall 1999. Massachusetts Institute of Technology.

Dikken, Marcel den 2003: Comparative Correlatives and Verb Second. CUNY Graduate center. New York.

Gadet, Françoise 1992: Le français populaire. Que sais-je? Paris: PUF.

Halmøy, Jane-Odile 1983: Le gérondif. Eléments pour une description syntaxique et sémantique. Tapir. Université de Trondheim.

Hansen, Åge 1967: Moderne Dansk I-III. København: Grafisk Forlag. Helbig/Buscha 1991: Deutsche Grammatik. Ein Handbuch für den Ausländerunterricht. Leipzig: Langenscheidt.

Jespersen, Otto 1924/1968: The Philosophy of Grammar. London: George Allen \& Unwin LTD.

Jespersen, Otto 1940/1952: A Modern English Grammar. Copenhagen: Munksgaard.

Korzen, Hanne 1992: The predicative unit and subject-verb inversion in modern French. Copenhagen Studies in Language 15. København: Handelshøjskolens Forlag. 
Lehmann, Christian 1988: Towards a Typologi of Clause Linkage. In Haiman, John/ Thompson, Sandra A. (Eds.), Clause combining in grammar and discourse. Amsterdam/Philadelphia: John Benjamins Publishing Company.

Quirk, Randolph/Greenbaum, Sidney/Leech, Geoffrey/Svartvik, Jan (1980): A Grammar of Contemporary English. London: Longman.

Riegel, Martin/Pellat, Jean-Christophe/Rioul, René 1994: Grammaire méthodique du français. Paris: PUF.

Sandfeld, Kristian 1965: Les propositions subordonnées. Genève: Librairie Droz.

Savelli, Marie-Josée 1993: Contribution à l'analyse macro-syntaxique: les constructions «siamoises» du type: «Plus V 1... Plus V 2...». Université d'Aix en Provence.

Savelli, Marie-Josée 1995: « Autant le dire ». Quelques éléments comparatifs sur la macro-syntaxe de plus/moins/autant. Recherches sur le français parlé ${ }^{\circ}{ }^{\circ} 13$. Aix en Provence.

Stage, Lilian 1991: Analyse syntaxique et sémantique de la conjonction si dans les propositions factuelles. Revue Romane 26, 2. Copenhague: Munksgaard.

Stage, Lilian. 2003a: Plus ça change, plus c'est la même chose. Om siamesiske konstruktioner på en række europæiske sprog. Copenhagen Working Papers in LSP. København.

Stage, Lilian 2003b: Siamesiske konstruktioner på en række europæiske sprog. SPRINT

Sproginstitutternes Tidsskrift Handelshøjskolen i København.

Stage, Lilian 2005 : Les constructions siamoises. Etude sur les comparatives corrélatives. XVI ${ }^{\mathrm{e}}$ Congrès des Romanistes Scandinaves [online]. www.ruc.dk/cuid/publikationer/publikationer/XVI-SRK-Pub/JUS/JUS06-Stage

Taylor, Heather Lee 2004: «Interclausal (co)dependency: the case of the comparative correlative. Michigan Linguistic Society Annual Meeting, University of MichiganFlint [online]. www.ling.umd.edu/ htaylor

Thiersch, C. 1982: «The harder they come... »: A note on the double comparative construction in English in Sprachtheorie und angewandte Linguistik, Festschrift für Alfred Wollmann. Tübingen: Günter Narr Verlag.

Zifonun, Gisela /Hoffmann, Ludger/Strecker, Bruno (1997): Grammatik der deutschen Sprache. Berlin, New York: Walter de Gruyter. 